\title{
Editorial
}

\section{Circuits and Systems for Wireless Sensing}

\author{
Xiongchuan Huang, ${ }^{1}$ Xiaopeng Yu, ${ }^{2}$ Chao Wang, ${ }^{3}$ and Hao Gao ${ }^{4}$ \\ ${ }^{1}$ Broadcom, San Diego, CA, USA \\ ${ }^{2}$ Zhejiang University, Hangzhou, Zhejiang, China \\ ${ }^{3}$ Institute of Microelectronics, A $^{*}$ STAR, Singapore \\ ${ }^{4}$ Eindhoven University of Technology, Eindhoven, Netherlands \\ Correspondence should be addressed to Xiongchuan Huang; coby.x.huang@gmail.com
}

Received 8 March 2017; Accepted 8 March 2017; Published 19 March 2017

Copyright ( 2017 Xiongchuan Huang et al. This is an open access article distributed under the Creative Commons Attribution License, which permits unrestricted use, distribution, and reproduction in any medium, provided the original work is properly cited.

The concept of Wireless Sensor Networks (WSN) emerged in the last decade, with various applications proposed. It was envisioned that the ubiquitous sensing and communication devices would soon be integrated into our daily life, to provide information and convenience wherever and whenever we need. However, to date, we have yet to see mass adoption of such applications, mainly due to the lack of viable wireless sensing devices, as well as the fragmentation of the data storage, computing, and networking infrastructure.

Recent advances in the field of artificial intelligence, big data, and cloud computing, as well as concurrent attempts in standardization in low-power wireless networking and security, are paving the way for the commercialization of WSN applications. With the commercial ecosystem getting in place and infrastructures becoming mature, the challenge lies in the research and development in low-cost, miniaturized, and self-sustaining circuits and systems, to realize sensing and wireless communication functionalities which are critical in the future WSN landscape.

In this special issue, the latest research efforts on circuits and systems for wireless sensing are presented. 10 research papers are published in this issue, covering a wide variety of topics including MAC and routing protocols for wireless sensor networks, integrated circuits, and devices for wireless sensors, as well as complete wireless sensor systems for real world applications. The highlights of these papers are summarized as follows.

"A 5 V-to-3.3 V CMOS Linear Regulator with ThreeOutput Temperature-Independent Reference Voltages" by
S.-F. Wang, Ming Chi University of Technology, Taiwan, presents a power management building block for wireless sensor nodes. The linear regulator designed and implemented in $0.18 \mu \mathrm{m}$ CMOS technology achieves less than $2.153 \%$ of voltage variation across temperature range from $-40^{\circ} \mathrm{C}$ to $120^{\circ} \mathrm{C}$ with a wide range of output current between 0 and $200 \mathrm{~mA}$. This regulator is especially suited for providing stable power supply for low-cost, small-size, and integrated wireless sensor nodes.

Another circuit building block for wireless sensor nodes is reported by Y. Zhang et al. in their paper "A 2 GSps, 8-Bit Folding and Interpolation ADC with Foreground Calibration in $90 \mathrm{~nm}$ CMOS Technology." In contrast to low-power, low data-rate wireless sensing applications, wireless security and monitoring applications require large data throughput, which in return require high speed data convertors. In this paper, an 8-bit folding and interpolation (F\&I) analog-to-digital converter (ADC) has been designed with $90 \mathrm{~nm}$ CMOS technology. This ADC achieves $2 \mathrm{GS} / \mathrm{s}$ sampling rate, $45.93 \mathrm{~dB}$ single-to-noise and distortion (SNDR), and $210 \mathrm{~mW}$ power consumption under $1.2 \mathrm{~V}$ supply. Foreground digital-assisted calibration technique is implemented to correct conversion error caused by circuit offset.

While CMOS technologies have been extensively used for miniaturized circuit implementation in wireless sensor nodes, other integrated circuit technologies are being developed rapidly, with unique features not offered by CMOS. In the paper "High Frequency InGaAs MOSFET with Nitride Sidewall Design for Low Power Application" by J. Mo et al., 
an InGaAs transistor has been reported for high frequency, low-power applications, such as radio transceivers in wireless sensor networks. The authors introduce a novel transistor design with nitride side-wall, achieving lower leakage current and parasitic capacitance, which is essential for low-power wireless sensing applications.

As the integrated circuit technology has greatly reduced the size of the circuit by more advanced compact integration, antennas continue to dominate the overall formfactor of wireless sensor nodes. To address this issue, a compact antenna design is reported by X. Chen et al. in their paper "Design of a Compact UWB Antenna with Triple Notched Bands Using Nonuniform Width Slots." When ultrawideband (UWB) systems are used in wireless sensing applications, because of wide bandwidth, they suffer from interference from other narrowband systems including WLAN (5.15-5.825 GHz), WiMAX (3.3-3.7 GHz), and ITU $\mathrm{X}$-band satellites $(8.025-8.4 \mathrm{GHz})$. The compact antenna presented in this work utilizes two pairs of quarter-wavelength nonuniform width slots embedded into the radiating patch and the ground plane to implement three notches at these interference frequencies, therefore improving the robustness of UWB wireless sensor networks.

Another interference rejection technique for wideband radio receivers is reported by $\mathrm{K}$. Ying et al. from Eindhoven University of Technology, the Netherlands. In their paper "A Nonlinear Transfer Function Based Receiver for Wideband Interference Suppression," the nonlinear characteristic of typical radio receiver is exploited to enhance, instead of degrade, the interference suppression of receivers. An adaptive nonlinear transfer function has been used to achieve interference suppression at the RF stage (close to the receiver input), which can reduce the power consumption of the following stages in the receiver, including the ADC. With this technique, robust and energy-efficient wireless communication channel can be established for sensor networks.

The energy consumption of wireless sensor networks is not only determined by the power consumption of the electronics, but also impacted by the communication protocols. Substantial amount of research has been carried out on effective communications of the wireless sensor network through MAC and routing algorithms. In "AHMAC: Adaptive Hierarchical MAC Protocol for Low-Rate Wireless Sensor Network Applications," A. I. Al-Sulaifanie et al. present an adaptive hierarchical MAC protocol with cross-layer optimization for low rate and large-scale wireless sensor networks. It combines the strengths of LEACH and IEEE 802.15.4 and achieves energy efficiency, selfconfigurability, scalability, and self-healing. The AH-MAC protocol is claimed to achieve eight times better energy efficiency while improving throughput compared to the LEACH protocol.

In terms of routing algorithms, Y. Chen et al. report a lifetime optimization algorithm in the paper "A Lifetime Optimization Algorithm Limited by Data Transmission Delay and Hops for Mobile Sink-Based Wireless Sensor Networks." The proposed algorithm is compared to 3 other algorithms (MCP_RAND, MCP_GMRE, and EASR) and it is proven to improve network lifetime and reduce average amount of node discarded data and average energy consumption of wireless sensor nodes.

Another routing algorithm is reported in "Balanced Transmissions Based Trajectories of Mobile Sink in Homogeneous Wireless Sensor Networks" by M. Akbar et al. Again working with mobile sink based routing strategies, this paper proposes two schemes for data gathering and in wireless sensor networks, one with random movements of mobile sink (RMS) and the other with defined movements (DMS). Results show that RMS performs better than DMS in terms of data collection from dense regions first and remaining afterwards, while, in terms of stability, DMS trajectory shows better performance.

In the paper "Affine-Invariant Geometric ConstraintsBased High Accuracy Simultaneous Localization and Mapping" by G. Hua and X. Tan, emphasis is placed on sensing the ambient environment using image sensors and computer vision algorithms. A new appearance-based loop-closure detection method for online incremental simultaneous localization and mapping (SLAM) using affine-invariant-based geometric constraints is proposed. With this technique, 2D imagery can be used instead of 3D graphics, and better performance is achieved when compared to the conventional bag-of-words based SLAM approaches.

Last but not least, a complete Internet-of-Things (IoT) solution for food safety tracking focusing on organophosphorus compound detection is presented in "A Mobile-Based High Sensitivity On-Field Organophosphorus Compounds Detecting System for IoT-Based Food Safety Tracking" by $\mathrm{H}$. Jin et al. It consists of a customized sensor front-end chip, LED-based light source, low-power wireless link, and coin battery, along with a sample holder packaged in a recycled format. An android-based application is also presented which makes the absorptiometer accessible via the Internet. High sensitivity is achieved by this organophosphorus compounds detection system which is comparable to commercial spectrophotometers.

It is exciting to present this special issue which collects the latest advances in circuits and systems techniques for wireless sensing applications. We hope the articles published in this issue will promote discussions and inspire new innovations in the academy and the industry.

\section{Acknowledgments}

We would like to thank all the authors for their contributions and the anonymous reviewers for their voluntary support and constructive critiques in making this special issue possible.

$$
\begin{array}{r}
\text { Xiongchuan Huang } \\
\text { Xiaopeng Yu } \\
\text { Chao Wang } \\
\text { Hao Gao }
\end{array}
$$




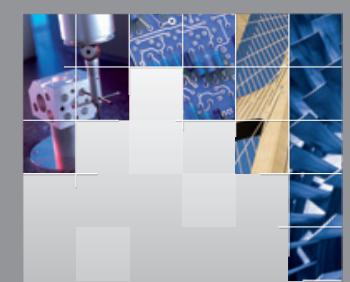

\section{Enfincering}
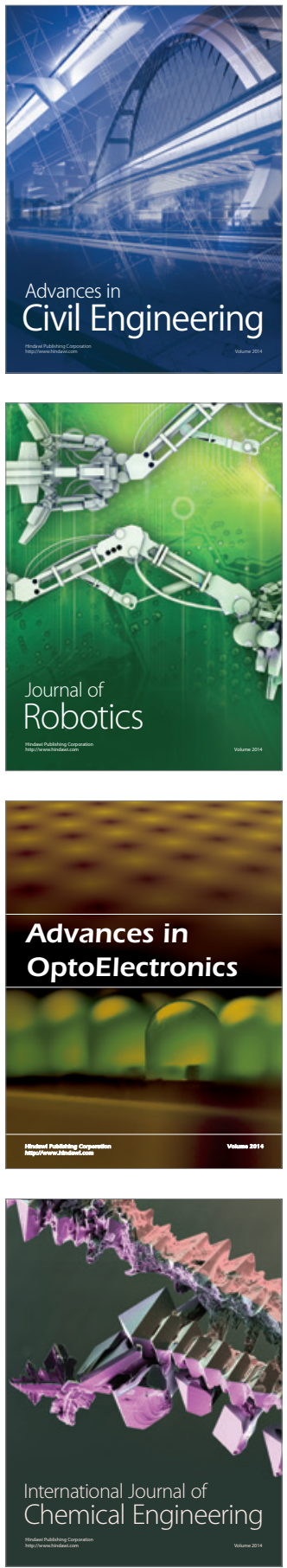

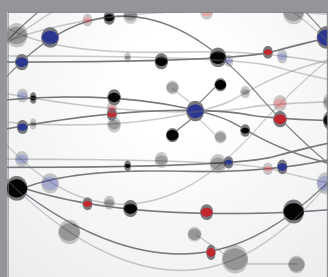

The Scientific World Journal

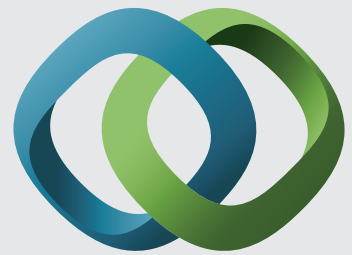

\section{Hindawi}

Submit your manuscripts at

https://www.hindawi.com
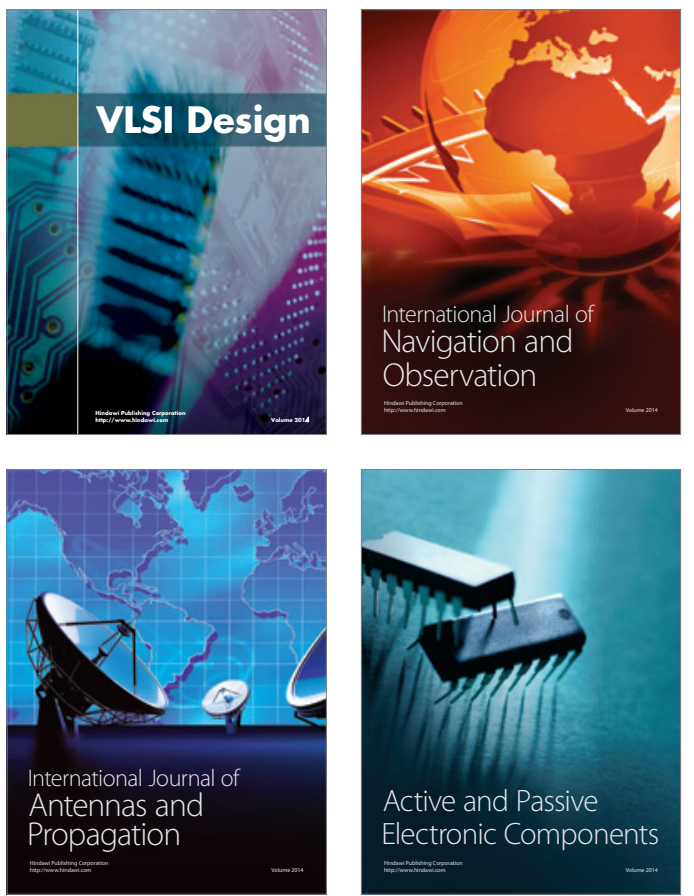
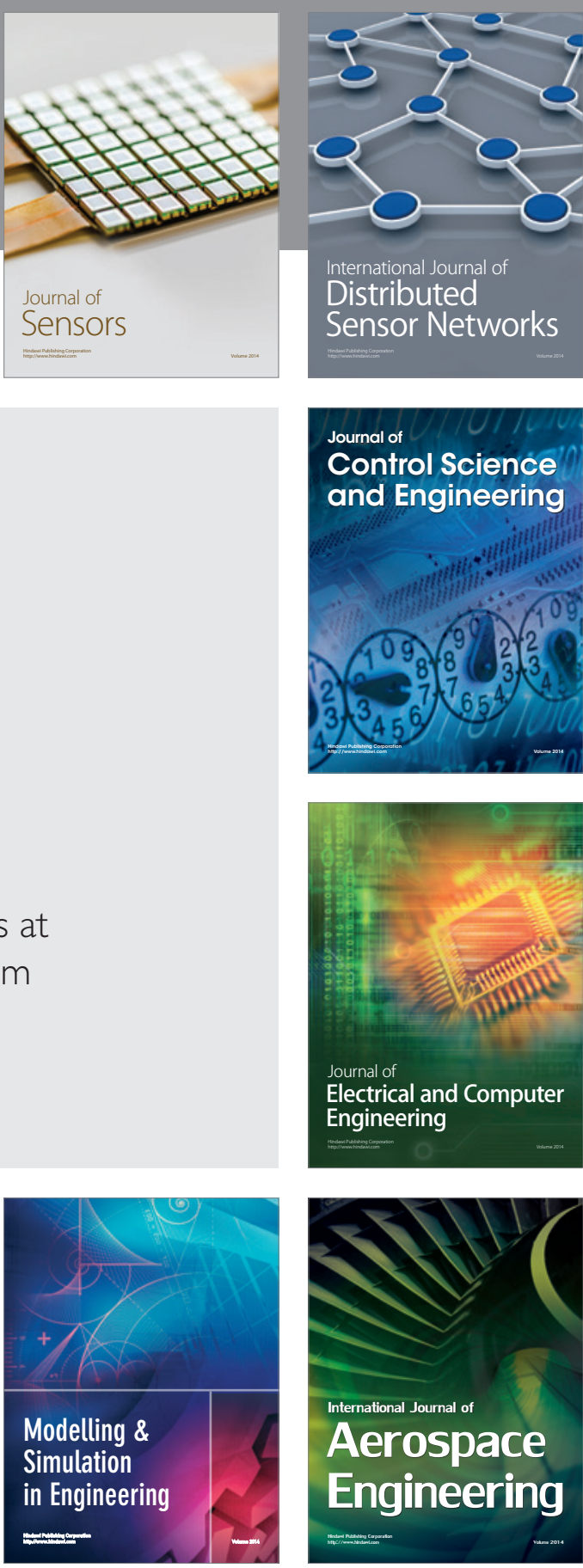

International Journal of

Distributed

Sensor Networks

$-$

Joumal of

Control Science

and Engineering
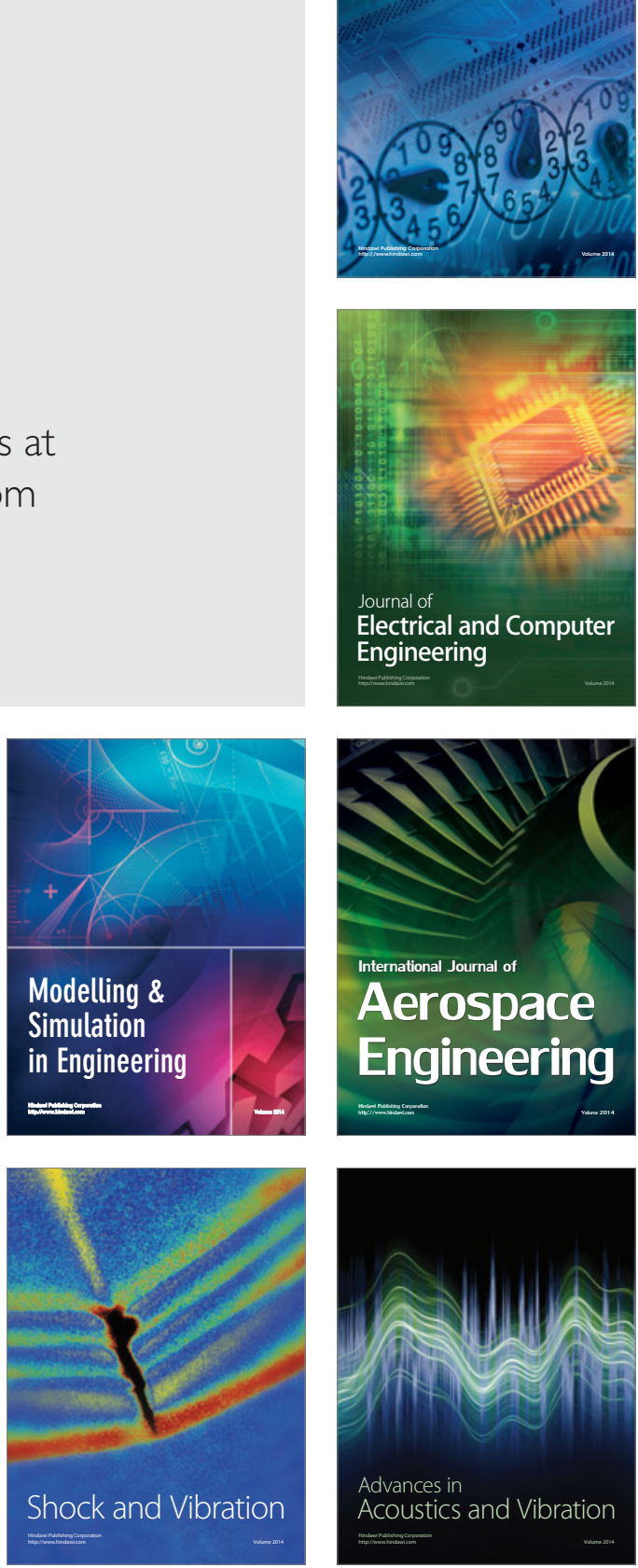